\title{
Gene-expression profiling is prognostic in ALL cases
}

Recent evidence from a study on children with acute lymphoblastic leukemia (ALL) has revealed that a set of novel gene-expression classifiers can identify the subgroup of children with a higher chance of relapse-free survival (RFS). This discovery, by Cheryl Willman and colleagues, might provide a reliable screening tool to identify children with high-risk ALL who do not benefit from current treatment regimens.

Treatment of pediatric ALL has improved considerably in the past decade; however, ALL relapse remains the second most common cause of cancer-related deaths in children in the USA. Currently, children with ALL are classified according to relapse-risk (categorized as low, intermediate, high, or very high) on the basis of minimal residual disease (MRD) measurements and pretreatment clinical characteristics. The investigators aimed to assess whether gene-expression profiling could be used to improve risk classification and outcome prediction, either alone or in combination with current techniques. "Gene-expression profiling has been one of the most powerful platforms for our development of molecular classifiers for outcome prediction in pediatric ALL, and for the discovery of novel therapeutic targets," says Willman.

The investigators obtained pretreatment leukemic cells from 207 children with B-precursor ALL diagnosed as being at high-risk of disease relapse based on MRD measures and pretreatment clinical characteristics. Through the use of gene expression microarrays, Willman's team correlated the expression of 23,775 genes with RFS in these children. Following robust statistical validation, a 38-geneexpression classifier was established that identified children at either low-risk (4-year RFS $81 \%$, 95\% CI $72 \%-87 \%$ ) or high-risk (4-year RFS 50\%, 95\% CI 39\%-60\%).

The researchers next combined the prognostic information from the 38-geneexpression classifier with that from flow cytometric measures of MRD, and found

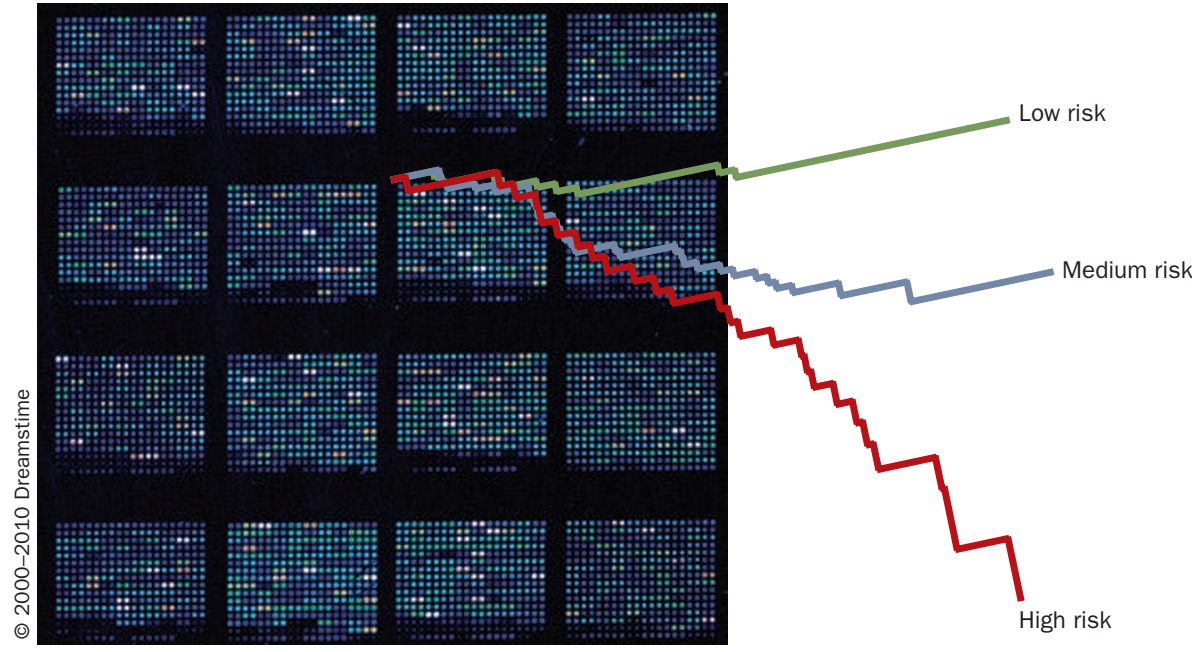

that application of the 38-gene-expression classifier further distinguished patients as either low-risk or high-risk with respect to disease relapse, irrespective of their classification based on MRD. In addition, further evaluation identified a 29-gene-expression classifier with similar prognostic potential in a subgroup of patients $(n=163)$ who lacked recurring cytogenetic abnormalities (another risk factor associated with ALL relapse). These results emphasize the increased prognostic power of this novel risk classification technique in comparison with current protocols in pediatric ALL.

In order to replace the prognostic utility of MRD measurements (which are restrictive because they are taken 29 days after the initiation of induction therapy), Willman and coworkers developed a 23-gene-expression classifier by assessing the relationship between gene expression and MRD in children with ALL. Combination of this 23-gene-expression classifier with the 38 -gene version for RFS resulted in a predictive tool that was capable of allocating children diagnosed with high-risk ALL into three distinct subgoups: low-risk, intermediate-risk, and high-risk (with 4-year RFS of $82 \%$, $63 \%$ and $45 \%$, respectively). Furthermore, the researchers validated the prognostic utility of these gene-expression classifiers in an independent cohort of 84 children with high-risk ALL from the CCG 1961 trial, who were also assigned into low-risk, intermediate-risk, and high-risk subgroups.

Interestingly, the extensive sequencing efforts involved in this study have lead to the discovery of novel JAK mutations in children with high-risk ALL, particularly those who have poor outcomes. Moreover, the gene-expression classifiers are capable of refining outcome prediction in the presence of JAK mutations, and thus the prognostic utility of the classifiers combined with the therapeutic potential of JAK inhibitors might be of benefit in pediatric ALL. "We have converted the gene-expression classifiers into a series of direct PCR assays on TaqMan ${ }^{\circledR}$ cards that can be more easily applied in the clinical setting," explains Willman. "We are now prospectively testing their utility to identify children who are not likely to respond to JAK inhibitors and other new agents in the future."

\section{Rowan Higgs}

